\title{
Explicit Modeling and Visualization of Imperfect Information in the Context of Decision Support for Tsunami Early Warning in Indonesia
}

\author{
Monika Friedemann, Ulrich Raape, Sven Tessmann, \\ Thorsten Schoeckel, and Christian Strobl \\ German Aerospace Center (DLR), German Remote Sensing Data Center (DFD), \\ Muenchner Strasse 20, 82234 Wessling, Germany \\ \{monika.friedemann, ulrich.raape, sven.tessmann, \\ thorsten.schoeckel, christian.strobl\}adlr.de
}

\begin{abstract}
A certainty model and appropriate visualization techniques are presented which are applied in a newly developed Decision Support System (DSS) for tsunami early warning deployed in Jakarta, Indonesia. Our decision support approach makes use of multi-sensor fusion and pre-computed tsunami scenario simulations to create situational awareness as basis for reasonable early warning. As the Indonesian coastline is prone to near-field tsunami scenarios decision making must take place under time-critical conditions based on incomplete and uncertain information. In order to reduce the probability and the consequences of a false decision, we have developed and employed a certainty model which implies a classification of imperfect information suitable for the tsunami early warning domain and the quantification of imperfect data. The model is mapped onto and supported by appropriate visual representations.
\end{abstract}

\section{Introduction}

The December 2004 tsunami demonstrated the need for an effective tsunami early warning system for the Indian Ocean. The work presented here is embedded in the German-Indonesian Tsunami Early Warning System (GITEWS) which has been operationally deployed in Jakarta as core of the Indonesian Tsunami Early Warning System (InaTEWS). The system combines a variety of sensor technologies such as terrestrial observation networks of seismology and geodesy, marine measuring sensors, satellite technologies, and pre-calculated tsunami scenarios used for multi-sensor online scenario selection by a Simulation System (SIM). The diverse sensor and simulation data is integrated, processed, and assessed by our Decision Support System (DSS) enabling the operator to make a concise assessment of the situation at hand and configure and disseminate the required warning messages [14].

What makes early warning for Indonesia unique and challenging is the extreme proximity of the coastline to the seismologically active Sunda Arc which initiates most of the tsunami incidents. A short time frame of merely half an hour is available for tsunami detection, warning, and evacuation increasing the operator's level of stress. Therefore, the generation of the best-possible situation awareness is crucial for a decision making by the operator at the earliest point in time. 
However, imperfection of information is common to early warning decision problems regarding sudden-onset types of disasters. Depending on the density of the sensor infrastructure, the types of sensors and communication networks used and the preprocessing required, sensor information will arrive in fragments, perhaps delayed, in arbitrary order and with initial uncertainties like pieces of a puzzle.

Research on the role of information imperfection for tsunami early warning is at its beginning. Academic research and projects driven by early warning centers in the Indian and Pacific Ocean focus on the tsunami propagation and inundation modeling and on error recognition and reduction in their forecast models [6, 10, 15]. Real-time tsunami modeling is in turn facing uncertainties and errors of real-time data sources which are used for making the tsunami forecast and deviations of the representing scenario to the reference data [15]. For the comparison of numerical and real data, some tsunami early warning systems provide therefore a display module where both time series are overlaid [1, 10, 15].

Indonesian operators in particular are forced to reason based on an incomplete and uncertain situational picture often without sufficient evidence from ocean sensors, in contrast to staff dealing with far-field tsunamis. The risk of late or false warnings which are expensive in terms of loss of warning credibility, economic loss, and even loss of lives is high. Our main motivation is therefore to optimize the tsunami early warning decision problem in terms of reducing late or false alarms by explicitly modeling and representing imperfect information throughout the decision support process. For this, we developed a certainty model which is used for three purposes:

1. Increase the operator's situation awareness (SA) and the confidence in it using information fusion and situation assessment techniques, including forecasting;

2. Based on 1., assess decision options and their consequences (costs) in order to propose the best option to the decision maker;

3. Based on 1. and 2., assess and propose the best point in time when to make the warning decision (trade-off between the pros and cons of "warn as soon as possible" and "decide as soon as enough information is available").

In the following, we will first outline the role of situation awareness in the decision support process. Afterwards, we will introduce our certainty model. We will conclude with supportive visualization techniques used throughout the process.

\section{Situation Awareness and Decision Support}

The main tasks of the InaTEWS DSS are to provide situation awareness to the operator and to support the early warning decision making process. To fulfill these tasks, concepts and methods of information fusion / sensor fusion and situation assessment/awareness have been applied while considering the specific requirements of human decision making under uncertainty and severe time constraints.

\subsection{Concepts of Information Fusion and Situation Awareness}

Two models of information fusion have been widely accepted. A functional definition is provided by the Joint Directors of Laboratories (JDL) which identify information 
fusion as the process of combining data to refine state estimates and predictions [8, 9, 13]. Endsley proposes another approach that addresses information fusion from a human perspective [4] which can be mapped to specific levels of the JDL model. Both approaches have been elaborated in detail in [11].

The Endsley model defines SA as the perception of elements in the environment within a volume of time and space, the comprehension of their meaning, and the projection of their status in the near future and therefore a constantly evolving picture of the state of the environment. Accordingly, the three main levels perception, comprehension, and projection have been identified which build the foundation for reasonable decision making and performance. During the remainder of the paper we will use Endsley's model which we adopted to the Tsunami Early Warning domain.

\subsection{Decision Support Process}

We use modeling result selections as another real-time input together with sensor information and a priori knowledge which are integrated and aggregated by different information fusion techniques to support the generation of situation awareness on all three levels. Thereupon an operator should be able to decide and act given a warning configuration for the event at hand. The cycle is repeated every time new information becomes available. Fig. 1 shows the process in a schematic way.

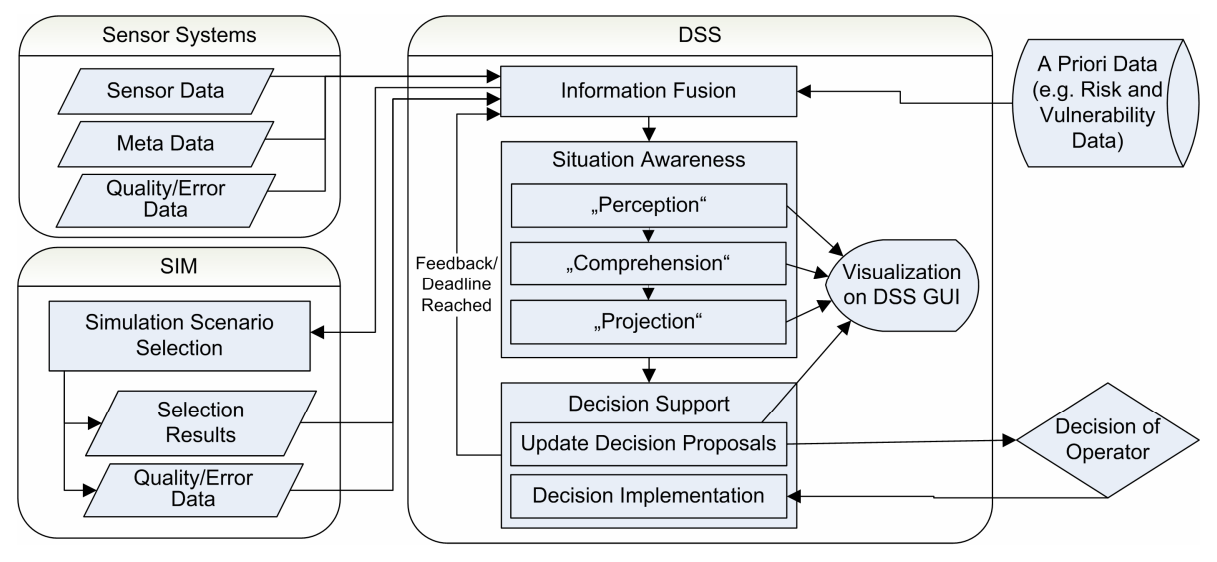

Fig. 1. The decision support core process. The system generates situation awareness based on the fusion of sensor data, modeling results, and a priori knowledge. Based on the generated SA, the system supports the operator to decide and implement the decision (decide and act).

The main task of the operator is thereby not just to warn as soon as possible risking false alarms, but rather optimize the early warning decision process under uncertainty. The early warning decision problem can be described as dynamic decision process which tries to minimize possible errors described in the following confusion matrix.

Both types of errors, false positive and false negative, produce costs such as casualties, economic losses and loss of confidence in the system, etc. In addition, the costs for a correct positive warning decision increase with time due to the decreasing time available for evacuation. 
Table 1. Confusion matrix for the classification of decision results

\begin{tabular}{lll}
\hline Situation Assessment & Tsunami & No Tsunami \\
\hline "Tsunami" Assessment & Correct Positive & False Positive \\
"No Tsunami" Assessment & False Negative & Correct Negative \\
\hline
\end{tabular}

Therefore, we extend the SA model by a planning level where the operator makes a trade-off between an immediate decision to warn or not to warn with sufficient confidence in the anticipated effects and the costs which would be caused by a wait decision to gain a clearer picture.

\section{Certainty Model}

We have developed a holistic model for imperfect information which we use throughout the SA generation process with the objective to promote more confident decisions. Besides providing a picture of the situation as complete and accurate as possible, we emphasize where the situation picture is imperfect. In order to serve a better comprehension of current facts and to facilitate projection into the future we take into account how data evolve over time and space.

\subsection{Taxonomy for Imperfect Information}

As a first step, we tried to identify all meanings of informational imperfection which are relevant to the tsunami early warning process. As we use a more-is-better pattern throughout our system which defines e.g. longer graphs and higher numbers as being more positive we decided to define terms of perfection instead of imperfection.

We combined the taxonomy presented in [12] with the types of uncertainty elaborated in [4]. As a result, we classify information perfection into precision, certainty, validity, consistency, and reliability.

Information is imprecise if it is incomplete, ambiguous or fuzzy whereas certainty relates to the knowledge of the truth of a statement. Denoting that "the earthquake has produced a tsunami of at least $1 \mathrm{~m}$ height and I' $\mathrm{m}$ sure about it" is imprecise but certain whereas "the earthquake has produced a tsunami of $3.5 \mathrm{~m}$ but I'm not sure about it" would be precise but uncertain. Both aspects can coexist but are distinct. Often any increase in one is balanced by a decrease in the other. We believe that certainty of the situational description is more crucial for a reasonable decision than uncertain precision since the latter facilitates unsubstantiated confidence.

Validity of information is determined by its timeliness. If data collection occurs continuously, information is mostly up-to-date. However, it is common to sensor technologies that data may arrive sporadically and delayed or may require preprocessing which reduces data validity. In addition, there is a connection between timeliness and certainty. Sporadic data updates introduce a degree of uncertainty as to what has changed in the interim.

Different data sources are consistent if they are in agreement on a phenomenon. InaTEWS DSS must be able to cope with inconsistent data. Imagine a tide gauge sensor measuring a significant wave traversal while an adjacent gauge sensor remains 
calm. Consistency can increase subjective confidence in the accuracy of the data. If multiple sources report conflicting information on the same situation confidence or certainty can be lowered and thus may have a negative effect on the decision process.

Uncertainty can be produced by low reliability of the sensors measuring the underlying phenomenon. Using our former example the second gauge sensor may have a minor reliability e.g. due to battery outage or ambient noise. Regarding tsunami detection, certain sensor types are inherently more reliable than others. The detection of an earthquake by the seismic system can indicate a tsunami but is less reliable regarding tsunami detection than ocean sensors which can actually prove a wave traversal.

\subsection{Fitness for Purpose Analysis}

Incoming data have heterogeneous attributes relating to some type of perfection. The purpose of information is to support decision making, and likewise the quality of information is dependent on the decisions, or the range of decisions, considered [2]. Using the classification above, we tried to identify relevant attributes provided by GITEWS input data. For instance, the list of tsunami scenarios selected by the SIM based on a given set of current sensor observations contains inherent precision and certainty in terms of list length and homogeneity. The better one or more scenarios match the measured situation distinctively the shorter the list. In addition, matching results are precise if the forecasts made by the scenarios in the list are homogenous, i.e. if all scenarios anticipate similar wave heights (estimated wave heights, EWHs) for all coastal points. In order to quantify the certainty of a tsunami scenario selection, the SIM provides a mismatch value as generalized distance of the scenario to the measured situation [3]. Other types of sensor observations data contain similar certainty information such as discrete error measures and confidence intervals. Regarding the reliability of a sensor each sensor is described by a number of characteristics among others a health code and a reliability value given by a sensor expert.

\subsection{Quantification of Perfection}

Based on the results of the fitness for purpose analysis the DSS quantifies the perfection data by a Quality and Certainty parameter which both are values in the unit interval. In the scope of the GITEWS DSS

- the Quality parameter describes the inherent perfection of information provided by sensors (both real sensors and SIM information before data fusion);

- the Certainty parameter describes the assessed perfection of fused information provided by multiple sensors in consideration of relations between the information.

Both parameters are currently generated using numerical methods. Quality is directly derived from perfection attributes such as the mismatch value provided by the SIM. The Certainty value is composed by fusion of individual Quality parameters and qualified by balancing factors regarding consistency and sensor reliability. The ability of sensor types to confirm a (tsunami) wave (e.g. ocean sensors) rather than only indicate the possibility that one was generated (e.g. seismology) is for instance weighted more strongly. Hence, the Certainty parameter bears - besides quantified perfection 
data - qualification assumptions which can be used to assess whether sufficient evidence is available for a warning decision. We differentiate levels of Certainty:

- Single-domain/single-source Certainty describes the fusion of Quality measures of different observations of one station.

- Single-domain/multi-source Certainty relates to fused Certainty information of different stations in order to assess the whole sensor system's outcome.

- Multi-domain/multi-source Certainty which we also call global Certainty is based on the fusion of Certainty information of different systems in order to assess the Certainty of the tsunami incident.

Both quantifiers can be mapped onto the SA levels. The Quality parameter is used to facilitate perception of inherent data perfection. On comprehension and projection level we make use of the Certainty value in order to quantify composite perfection, to enable comparison of sensor system outcome on an abstract level, and to make assumptions regarding potential trends. A detailed description of the mapping functions is outside the scope of this paper and will be covered by subsequent publications.

\section{Visualization of Imperfect Information}

Many of our design decisions are based on commonly accepted user interface principles such as overview first, details on demand, on the internationally standardized dialogue principles for interactive systems (ISO 9241-110) [7] and on Endsley's design principles for increasing SA [5]. Some of those SA principles address the representation of uncertainty and the increase of confidence in the context of decision support [4]. This paper concentrates on presentation techniques and patterns which reveal the perfection of the situation picture on all levels of SA.

\subsection{Perception Visualization}

Perception visualization provides representations of the status, attributes, and dynamics of the relevant elements as basis for the higher levels. A first SA principle is employed by explicit identification of important missing information by color, e.g. colored cell in table or colored sensor station in map. In addition, sensor reliability is presented by different types of sensor status icons. Fig. 2 shows a map extract with a gray thus offline (i.e. no data expected) buoy station next to a white thus active buoy station in tsunami mode (i.e. frequent data updates expected) and a pale-red crossedout tide gauge station which reports invalid information (upper left). The table extract below shows marked missing wave height information of the gauge station in detail.

The quantifiers Quality and Certainty are mapped directly on a bar which is subdivided into fixed blocks which we believe leads to faster perception compared to numerical percentages or analog bars. Certainty bars are shown in Fig. 3.

Another SA design principle comprises the representation of information timeliness in order to assess validity of those information. We have implemented a newest-on-top principle by which the most current information is placed at the top of each information module. Upon update the respective data set (e.g. collection of measurements of a 
sensor) moves to its new position indicating risen sensor validity. A similar principle is used with the emphasis of "idle" time since the last incoming measurement in DSS time series modules. The DSS displays sea level measurements of tide gauges and buoys over time. As shown in Fig. 2 (right) the time range for which measurements exist is highlighted gray which we define as color representing the past. The time since the last collected measurement to a red now-line is marked white which makes it easier for the operator to identify which sensors produce timely thus valid information. In addition, the DSS decides which time series are displayed treating invalid sensors by default with lower priority and preferring valid sensors.
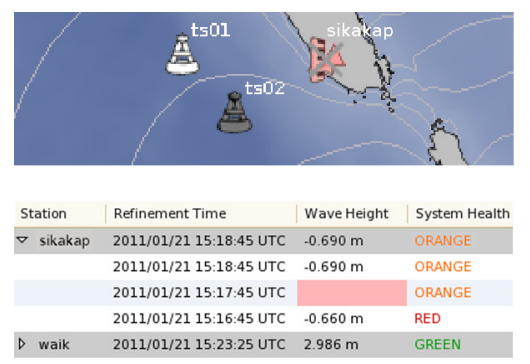

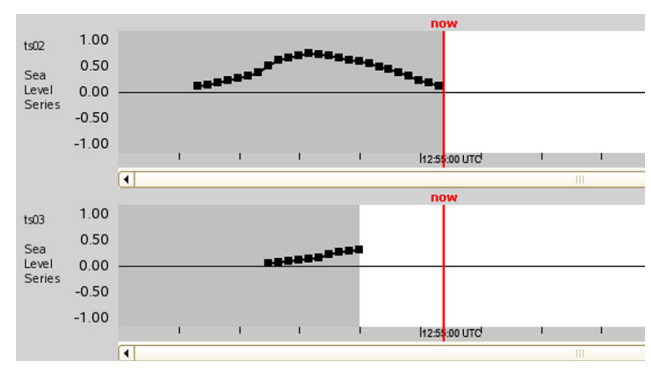

Fig. 2. Extracts of visualization modules on perception level. Map shows different types of sensor status icons, table cells with missing information are colored pale-red (left). Timeliness of sensors is emphasized by marking the time range since the last measurement white (right).

\subsection{Comprehension Visualization}

Comprehension includes the integration of multiple pieces of information and a determination of their relevance for the decision process in order to produce a composite picture. It takes primarily place by comparison of fused data. For this purpose, we intentionally stack the Certainty bars of all participating sensor systems (singledomain/multi-source Certainty) and disregard any details as shown in Fig. 3 (left). Each Certainty bar relates to the certainty of the underlying phenomenon, such as the certainty of the assessed earthquake severity (Earthquake Monitoring System) or the certainty of the assessed sea level anomaly (Tide Gauge System). By this arrangement ambiguities can be faster identified and sensor systems with incomplete or inaccurate information are discretely emphasized which conforms to another SA design principle. Based on this high-level interpretation the user can selectively explore uncertainty information in detail by analyzing the small-scale information in tables and in visualization modules to build confidence in composite data.

An example for the investigation of imperfection in composite data is provided by a graph visualization of tsunami scenario selection results shown in Fig. 3 (right). The filled graph shows the EWHs (y-axis) for all affected segments at the coastline (x-axis) based on an aggregation of the list of selected scenarios. The overlaid thicker graphs represent the EWHs of the single scenarios in the list. By combining single and aggregated results in one diagram the operator is able to assess the homo/heterogeneity of the result list and thus the perfection of the matching. 

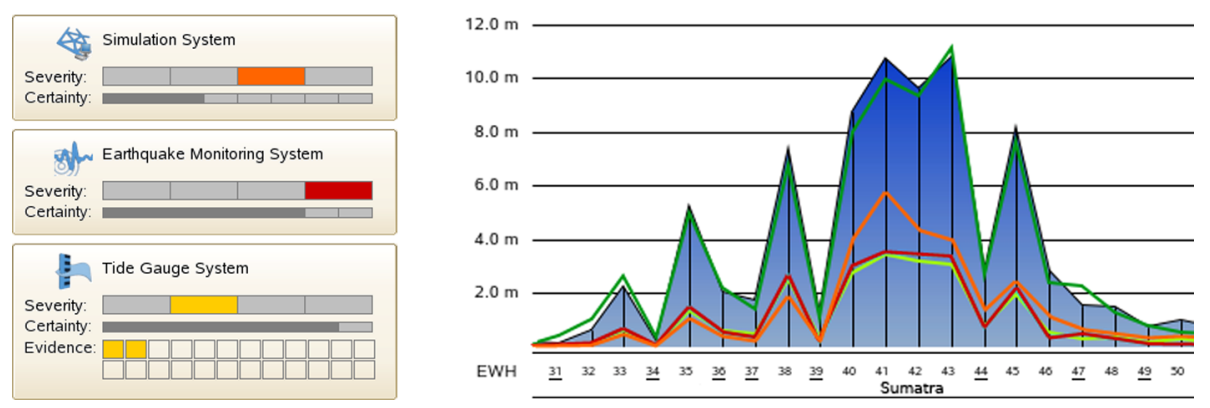

Fig. 3. Extracts of visualization modules on comprehension level. Stacked Certainty bars of different sensor systems are used for comparison on an abstract level (left). Heterogeneity of scenario selection result list can be explored by overlaying single scenarios of list (right).

Time plays an important role for the comprehension of data convergence such as the leveling-off of the earthquake magnitude value. A plot shows magnitude refinement evolution over time which helps the operator to assess the stability of the current knowledge and thus also sensor reliability. As used by other tsunami early warning systems the overlay of different time series such as simulated and real-measured sea level recordings helps understanding their consistency.

\subsection{Projection and Planning Visualization}

A critical part of SA is understanding how much time is available until some event occurs or some action must be taken. Time series modules and plots such as the magnitude refinement plot mentioned before help the operator to anticipate further evolution (e.g. convergence of the magnitude onto a stable value) and to schedule actions.

For the overview of the course of events and actions on a temporal scale the DSS provides a timeline module (see Fig. 4 for an extract) which is separated from left to right into past, present and future. A red now-line represents the present point in time. Left of it sensor measurements are displayed on the lowest lane. On the lane above ("Simulation Forecast") representations of anticipated events such as the estimated wave arrival at a sensor station are placed. Important deadlines are shown as thick blue lines. A light-blue area defines the time from now to the next important deadline.

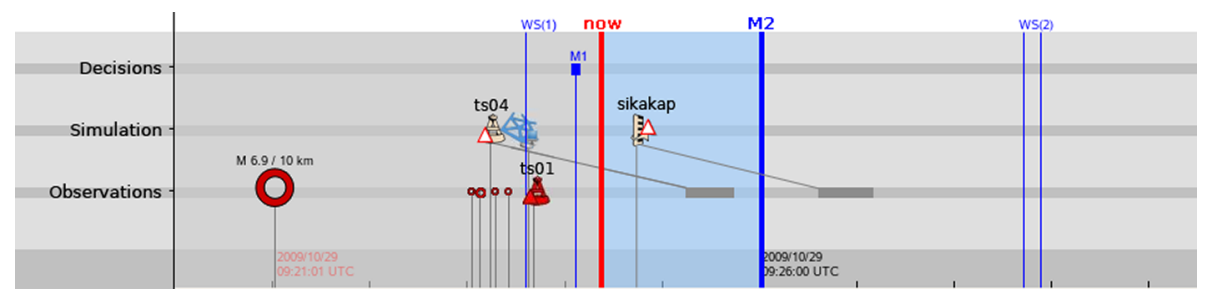

Fig. 4. Timeline visualization of sensor observations (icons on lowest lane), moving now-line (thick red line), important deadlines (thick blue "M2"- line), anticipated sensor observations and expected data arrival (sensor icons on middle lane and connected gray blocks on lower lane) 
By examining if any buoy or gauge station is expected to be reached shortly by the wave the operator can decide whether to wait for the likely sensor data update and substantiate his knowledge or to accelerate a warning action. In the example it would make sense for the operator to wait for additional evidence from buoy $t s 04$ since data arrival is expected to happen before the dissemination deadline for warning message "M2" will be reached. In contrast, it could be reasonable to ignore tide gauge sikakap which is expected to provide recordings after the deadline.

\section{Outlook}

The paper describes the certainty model and related visualisation aids of the current operational InaTEWS installed in Jakarta, Indonesia. A number of additional mapping functions and visualisation tools are under development which will provide further decision support to the operator, among them probabilistic forecast models, improved time-dependent trade-off / cost functions and certainty-based consistency metrics.

Acknowledgments. GITEWS has been developed by scientists and engineers of the German Research Centre for Geosciences (GFZ, Project Lead), the Alfred Wegener Institute for Polar and Marine Research (AWI), the German Aerospace Center (DLR), the GKSS Research Centre (GKSS), the German Marine Research Consortium (KDM), the Leibniz Institute of Marine Sciences (IFM-GEOMAR), the United Nations University Institute for Environment and Human Security (UNU), the German Society for Technical Cooperation (GTZ), the Federal Institute for Geosciences and Natural Resources (BGR) as well as Indonesian and international partners. The project is funded by the German Federal Ministry for Education and Research (BMBF), Grant 03TSU01.

\section{References}

1. Annunziato, A.: The Tsunami Assessment Modelling System by the Joint Research Centre. Science of Tsunami Hazards 26(2), 70-92 (2007)

2. Arnborg, S., Brynielsson, J., Artman, H., Wallenius, K.: Information Awareness in Command and Control: Precision, Quality, Utility. In: Proceedings of the Third International Conference on Information Fusion (FUSION 2000), vol. 2, pp. pp. THB1/25-THB1/32 (2000)

3. Behrens, J.: On uncertainty and quality control in tsunami forecasting. In: Proceedings of the Indian Ocean Tsunami Modelling Symposium, Fremantle, Australia, pp. 14-18 (2010)

4. Jones, D.G., Bolté, B., Jones, D.G.: Designing for Situation Awareness. Taylor and Francis, Boca Raton (2003)

5. Friedemann, M., Raape, U., Tessmann, S., Schöckel, T., Sparwasser, N., Hochleitner, G., Strobl, C.: Decision Support Interface for Tsunami Early Warning in Indonesia - Dealing with Information Fusion, Uncertainty, and Time Pressure. In: Proceedings of IHCI 2010, Freiburg, Germany, pp. 289-294 (2010)

6. Furumoto, A.S., Tatehata, H., Morioko, C.: Japanese Tsunami Warning System. Science of Tsunami Hazards 17, 85-105 (1999)

7. DIN EN ISO 9241-110: Grundsaetze der Dialoggestaltung. Beuth Verlag, Berlin (2006) 
8. Klein, L.A.: Sensor and Data Fusion: A Tool for Information Assessment and Decision Making. SPIE Society of Photo-Optical Instrumentation Engineering (2004)

9. Liggins, M.E., Hall, D.L., Llinas, L.: Handbook of Multisensor Data Fusion: Theory and Practice (Electrical Engineering \& Applied Signal Processing). CRC Press, Boca Raton (2008)

10. Nayak, S., Kumar, T.S.: Indian Tsunami Warning System. In: The International Archives of the Photogrammetry, Remote Sensing and Spatial Information Sciences, Beijing, vol. 37, part B4, pp. 1501-1506 (2008)

11. Salerno, J.: Information Fusion: a High-level Architecture Overview. In: Proceedings of the Fifth International Conference on Information Fusion, vol. 1, pp. 680-686 (2002)

12. Smets, P.: Imperfect Information: Imprecision - Uncertainty. In: Uncertainty Management in Information Systems, pp. 225-254 (1996)

13. Steinberg, A. N., Bowman, C. L., White, F. E.: Revisions to the JDL Data Fusion Model. Presented at Joint NATO/IRIS Conference, Quebec City, Canada (1998)

14. Steinmetz, T., Raape, U., Teßmann, S., Strobl, C., Friedemann, M., Kukofka, T., Riedlinger, T., Mikusch, E., Dech, S.: Tsunami Early Warning and Decision Support. Natural Hazards and Earth System Sciences 10, 1839-1850 (2010)

15. Titov, V., Mofjeld, H., Newman, J., Venturato, A., González, F., Bernard, E., Eble, M.: Real-time Tsunami Forecasting: Challenges and Solutions. Natural Hazards 35, 41-58 (2005) 